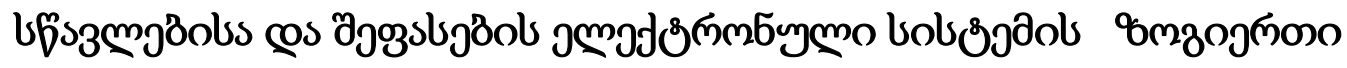

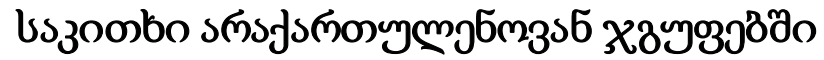

\author{
Rmbsmsdo asmosa

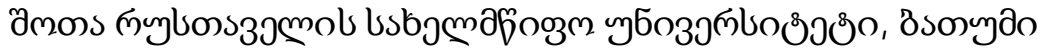 \\ https://doi.org/10.52340/idw .2021.545
}

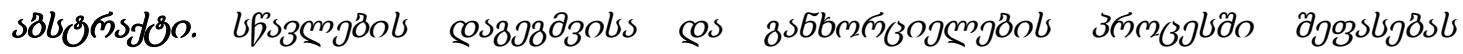

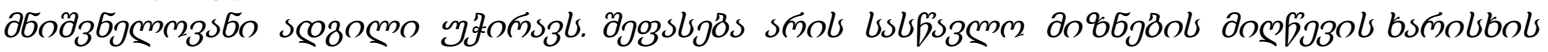

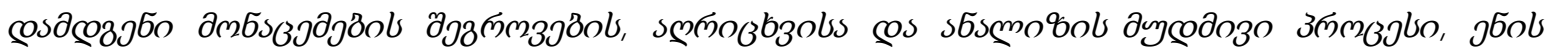

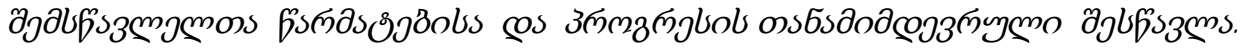

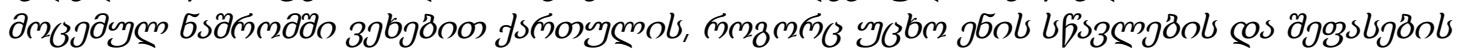

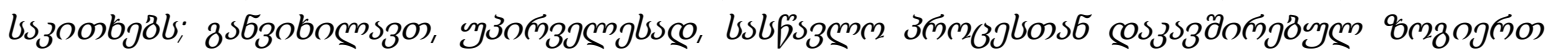

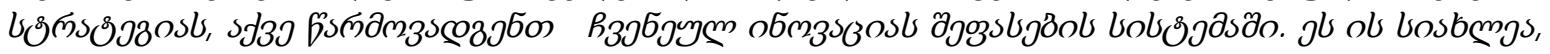

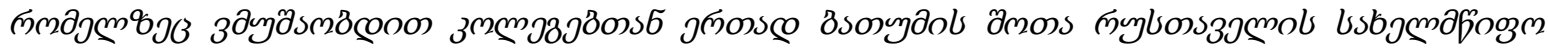

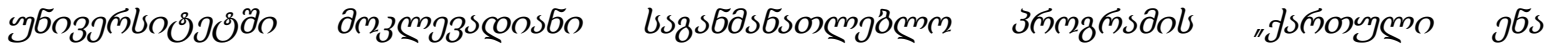

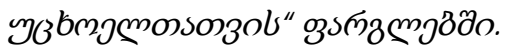

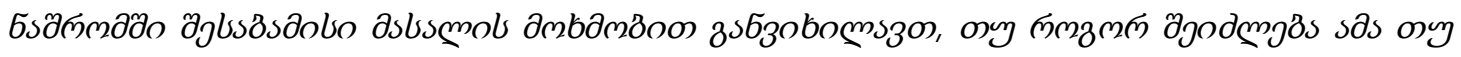

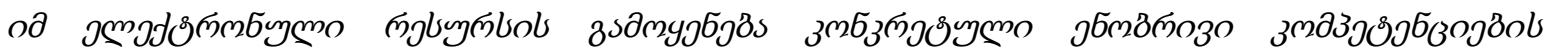

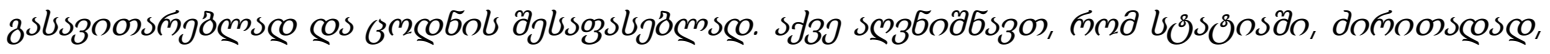

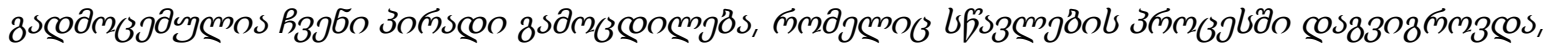

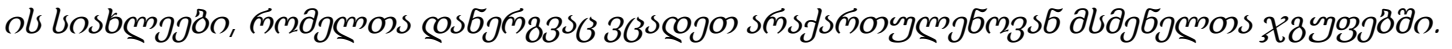

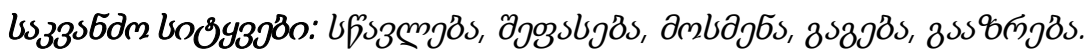

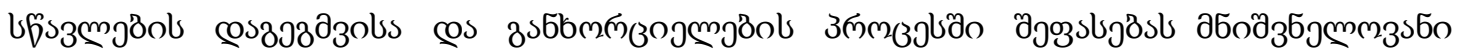

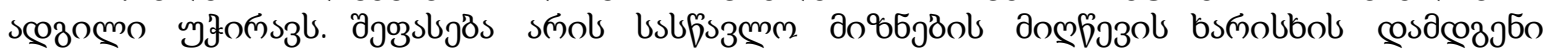

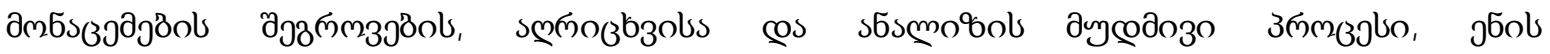

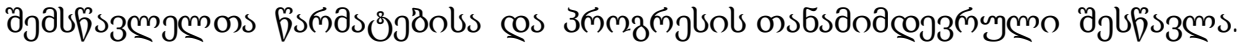

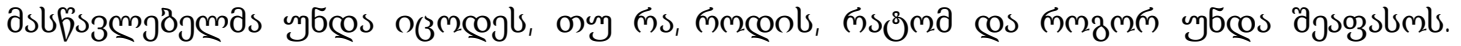

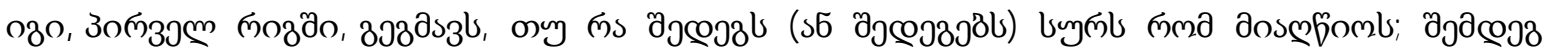

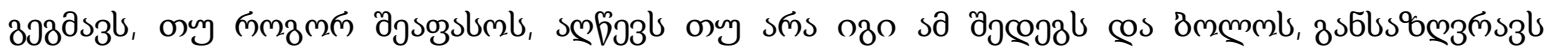

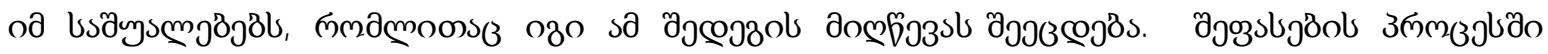

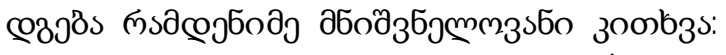

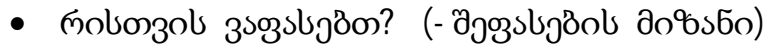

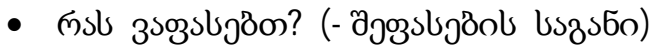

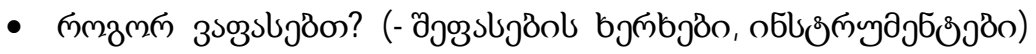

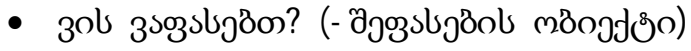

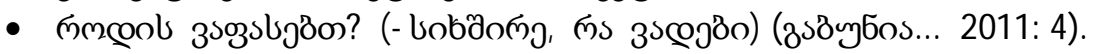

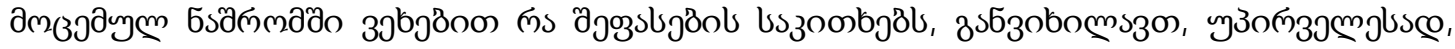

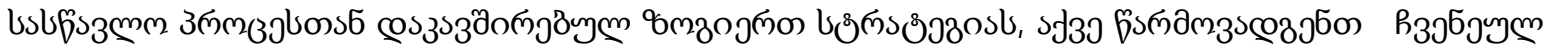

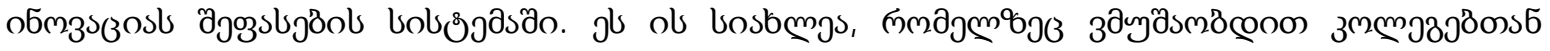

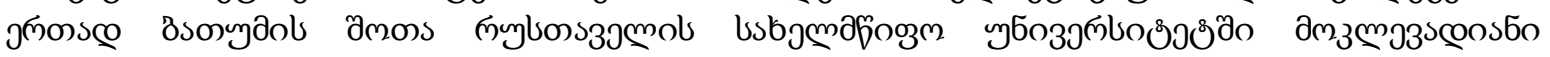
uszsбдsбsom 


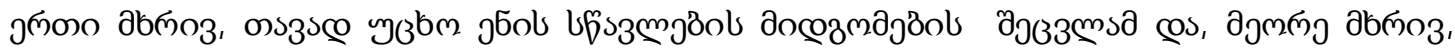

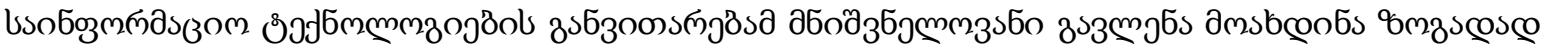

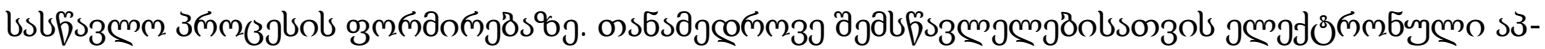

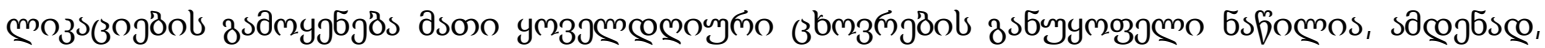

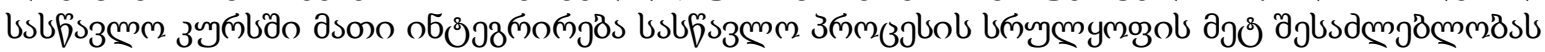
odmo3s.

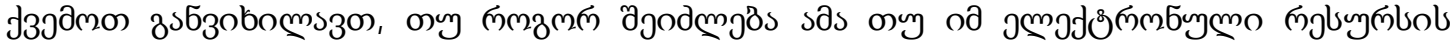

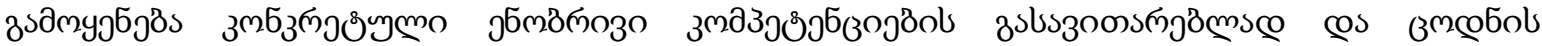

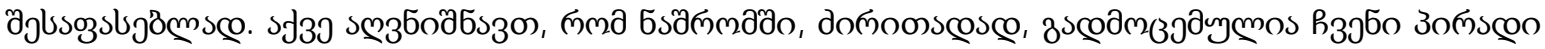

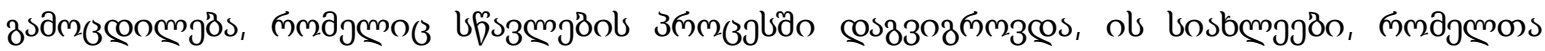

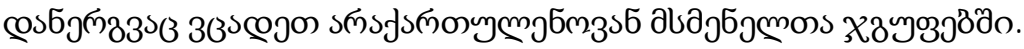

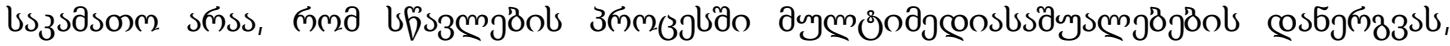

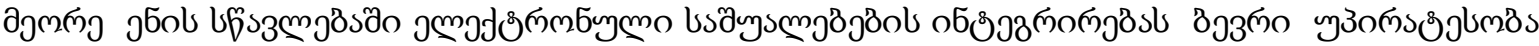

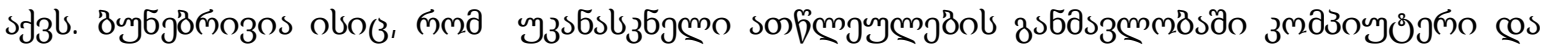

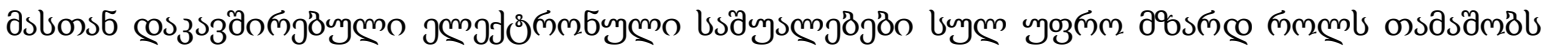

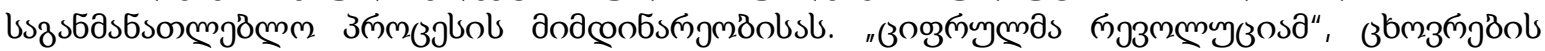

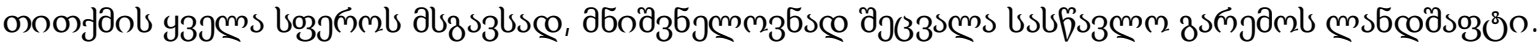

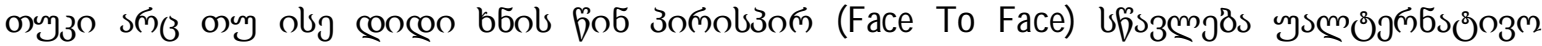

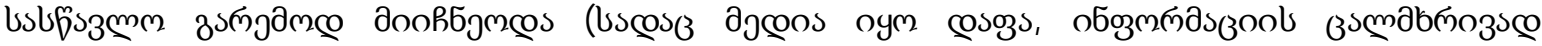

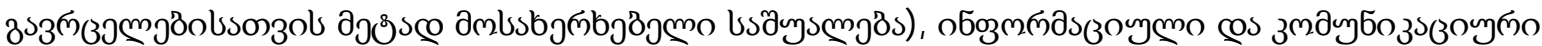

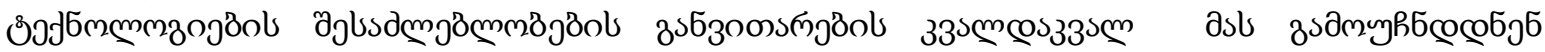

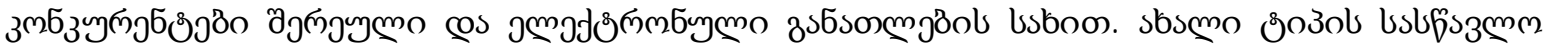

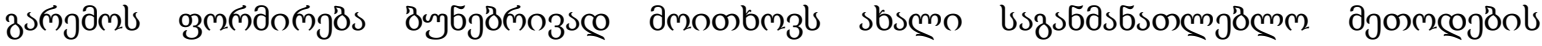

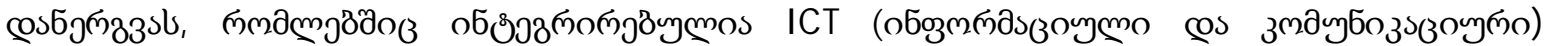

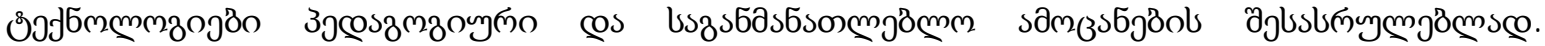

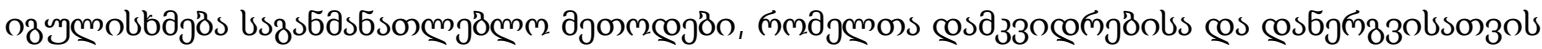

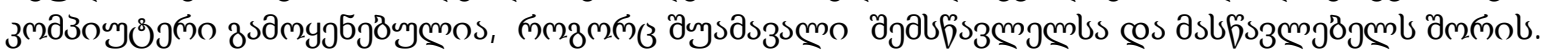

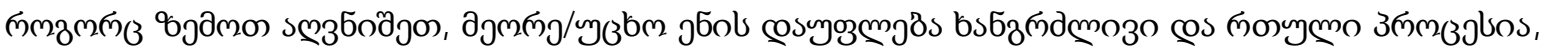

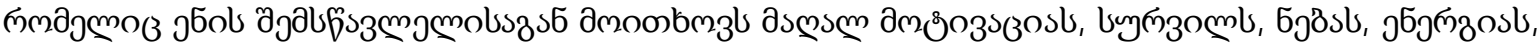

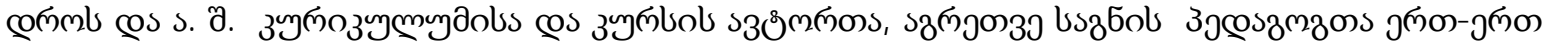

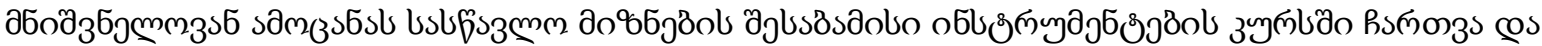

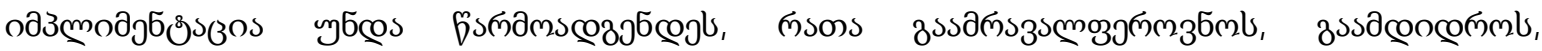

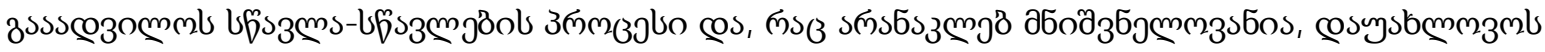

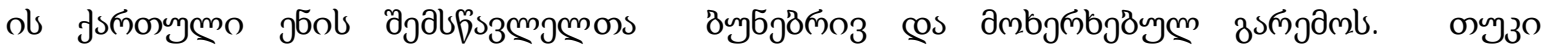

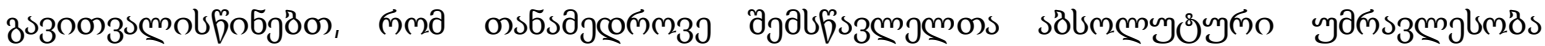

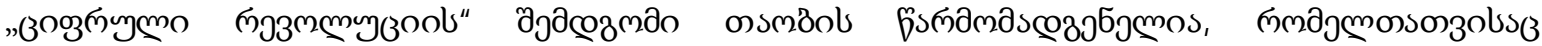

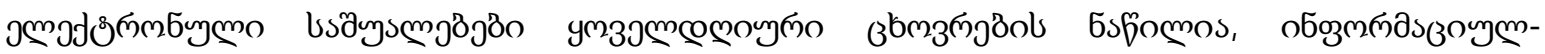

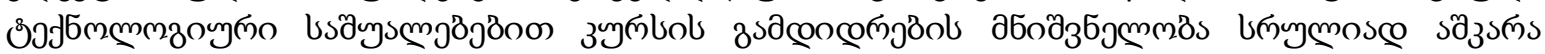

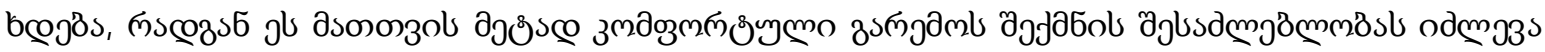

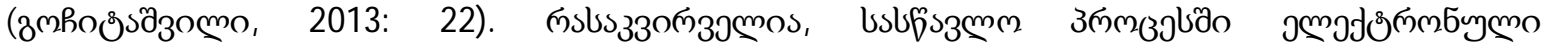

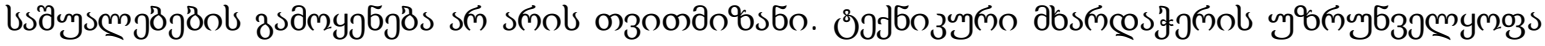

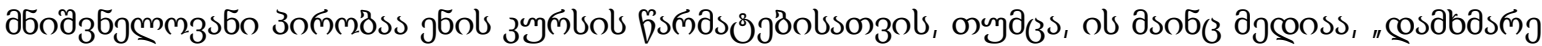

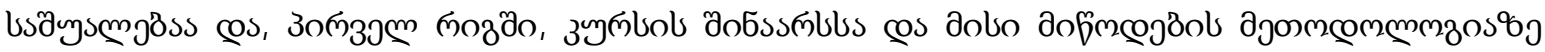

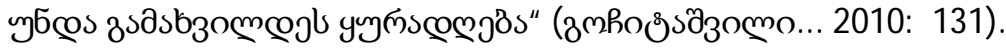

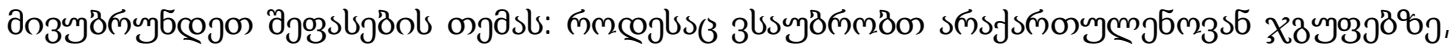

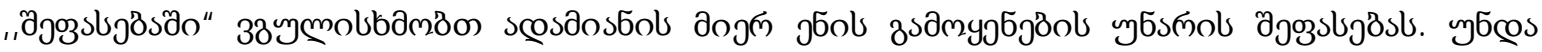

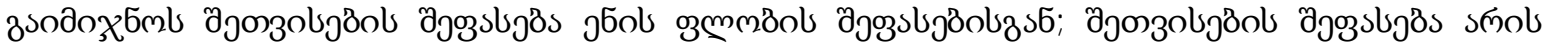
дып

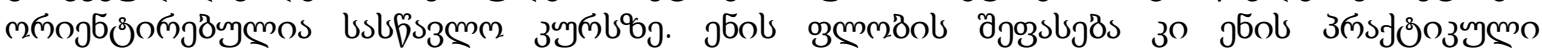

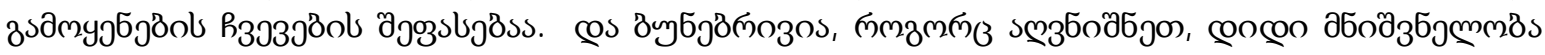




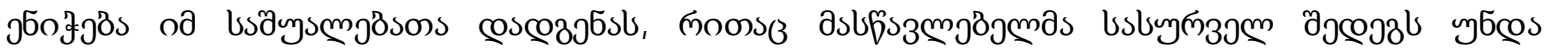
doschomb.

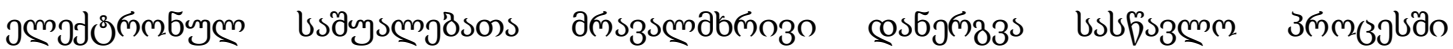

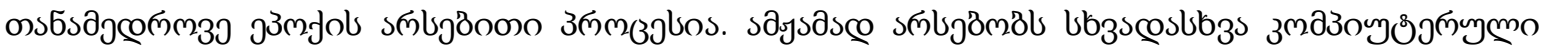

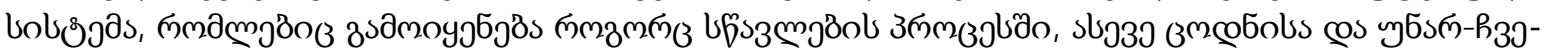

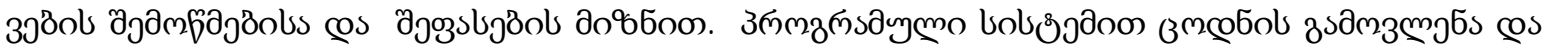

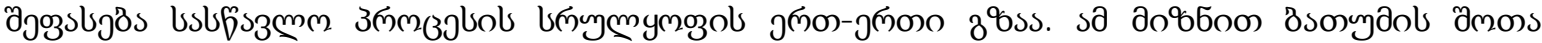

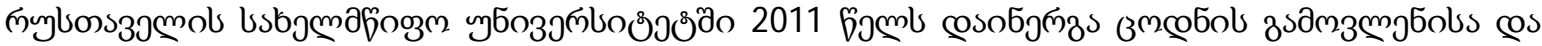

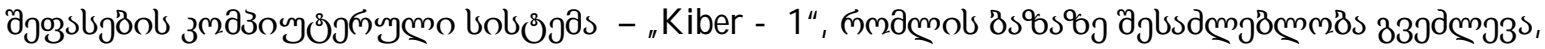

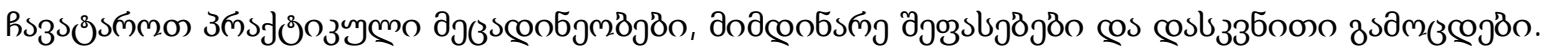

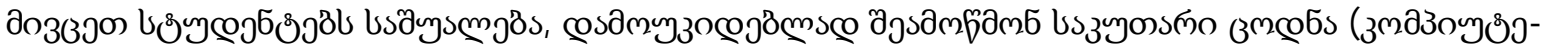

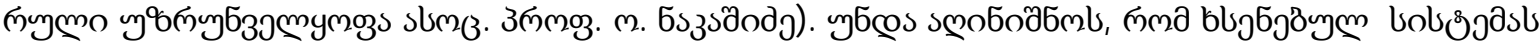

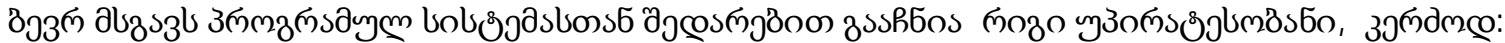

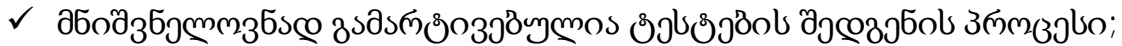

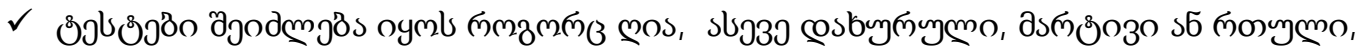

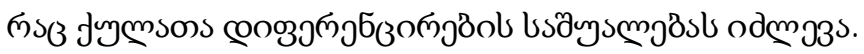

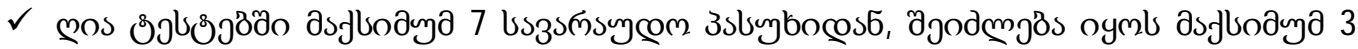
ufmепо 3sluybo;

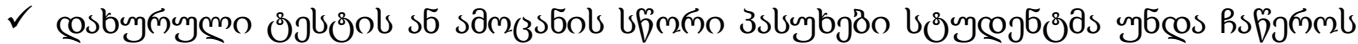

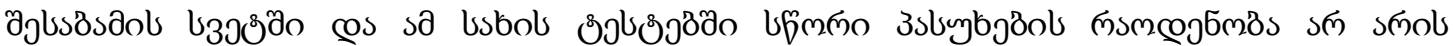

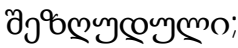

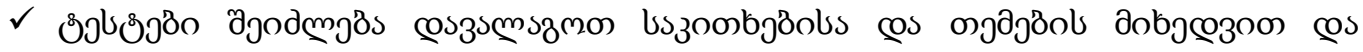

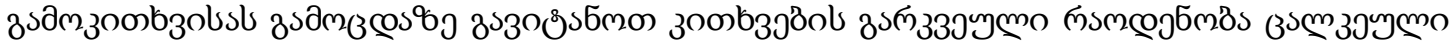
ojaob dobวœзом;

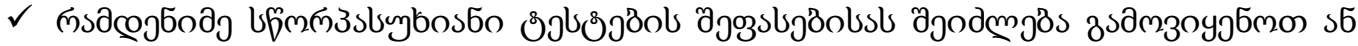

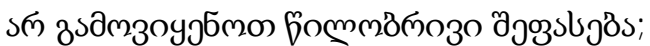

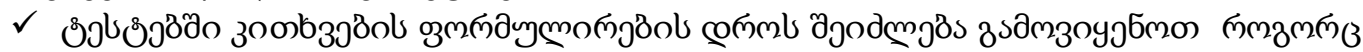

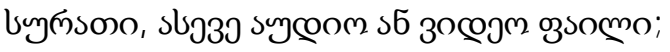

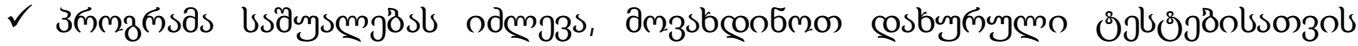

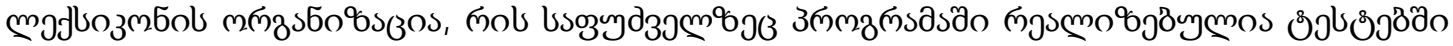

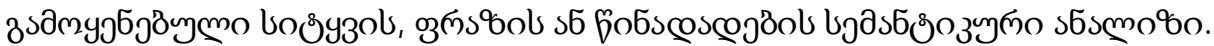

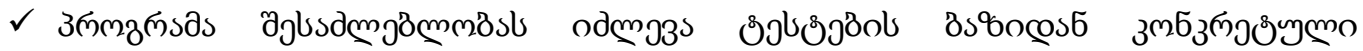

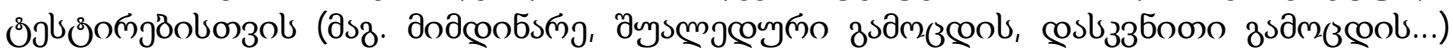

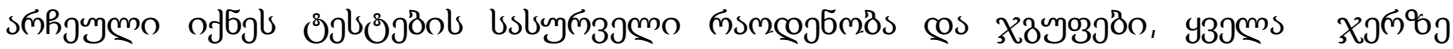

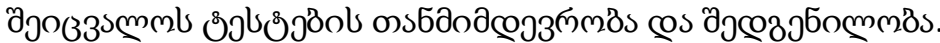

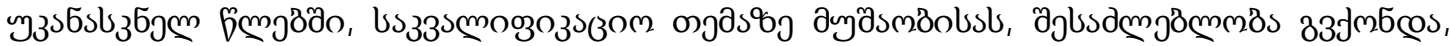

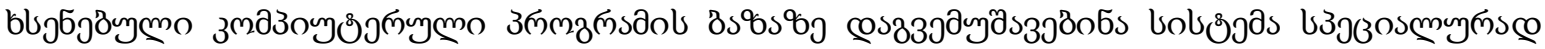

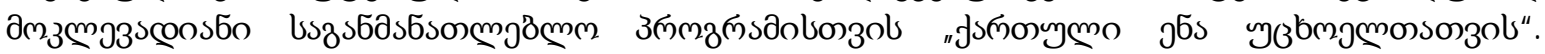

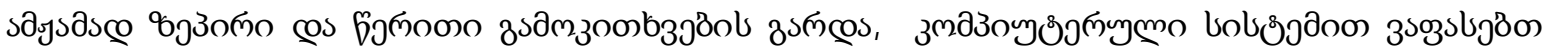

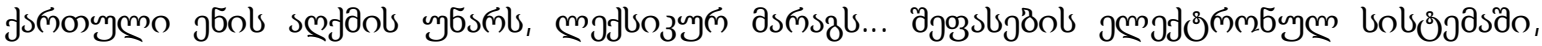

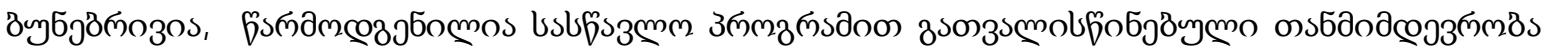

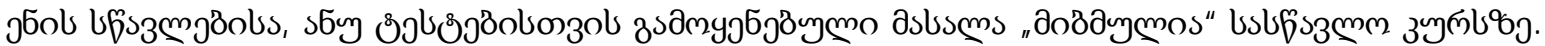

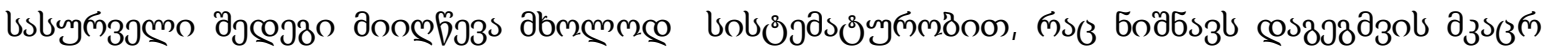

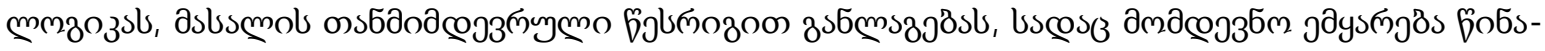

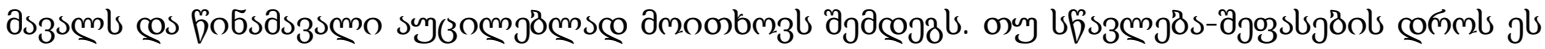

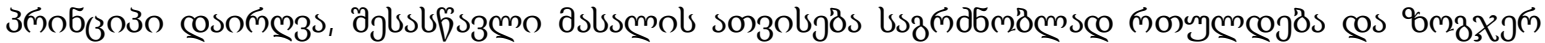

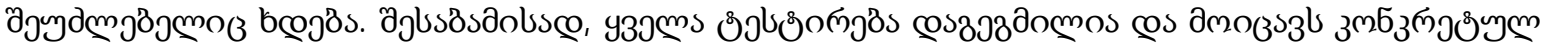

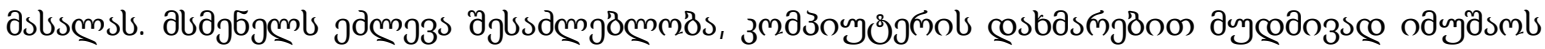

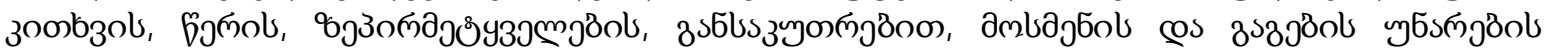

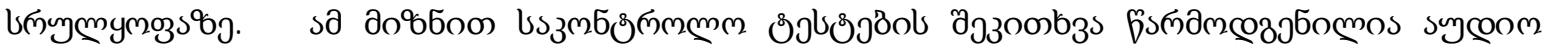




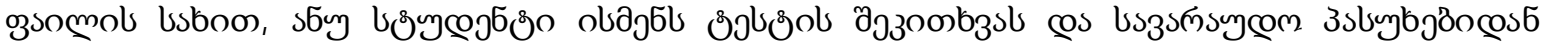

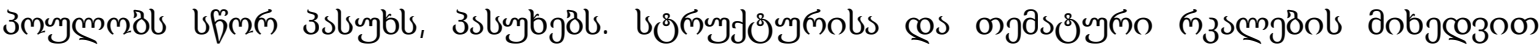

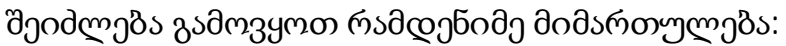

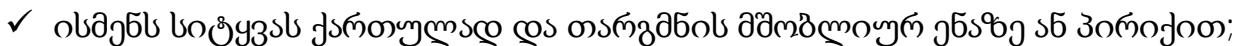

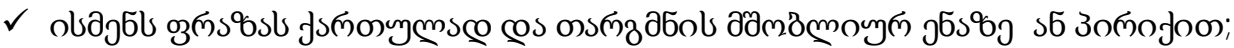

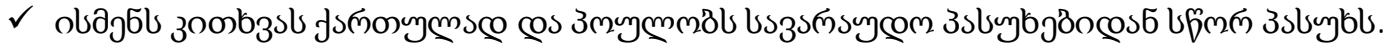

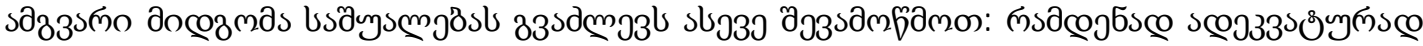

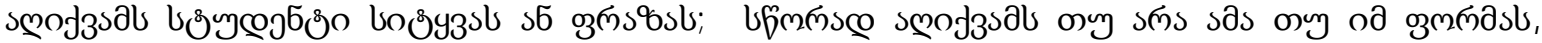

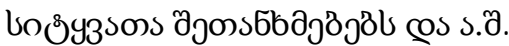

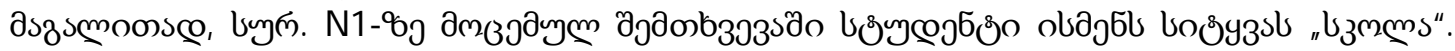

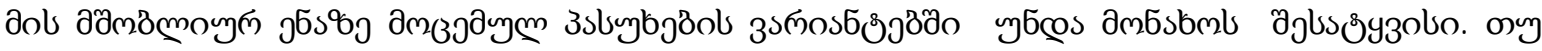

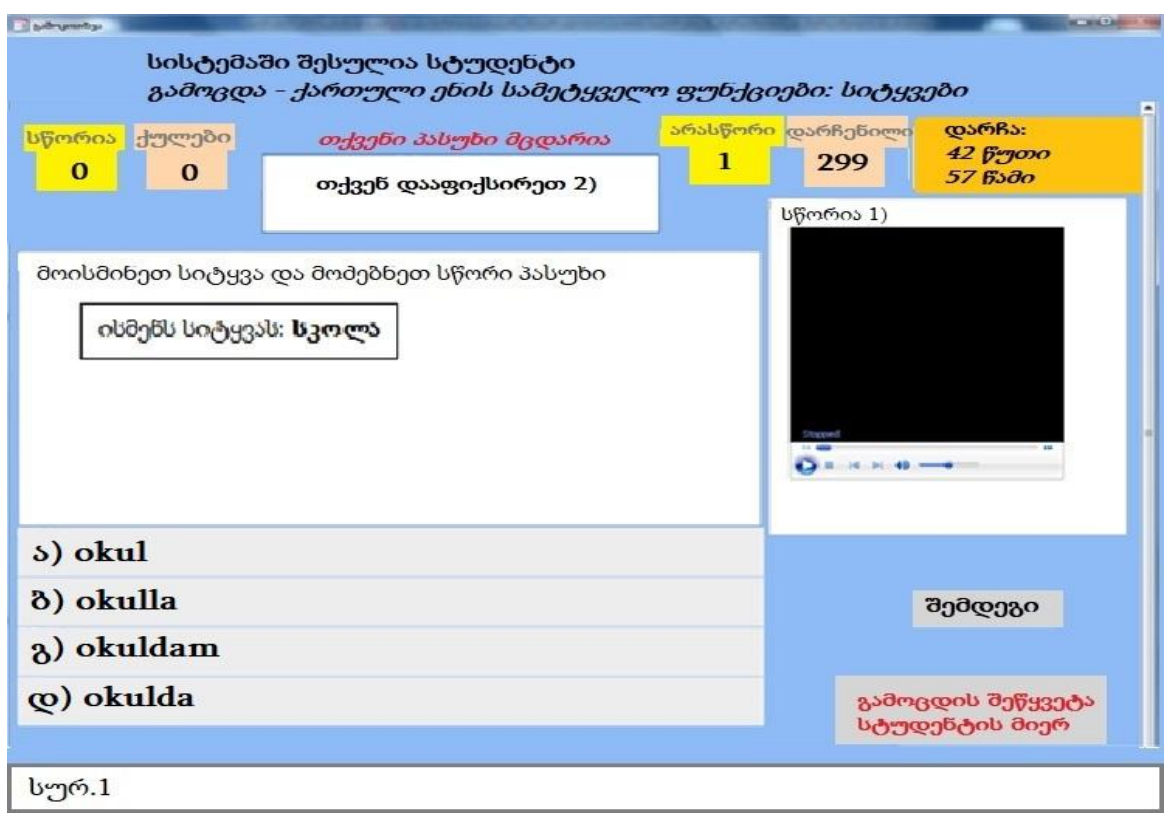

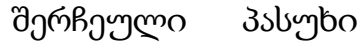
sल smols ufmmo, bolơjas samo3ls

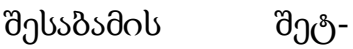
ymß̈oбjßsts.

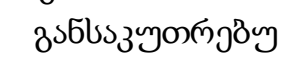

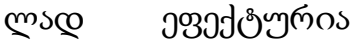

дмзәдулмо

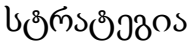

moflozs\%

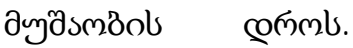

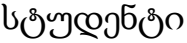

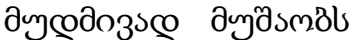

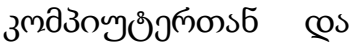

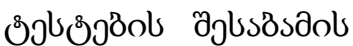
Bsostrons6.

moflozymo дsmszol ongool 33s

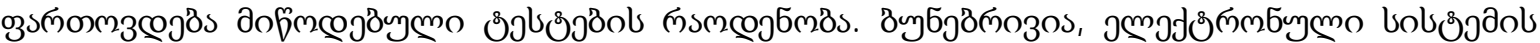

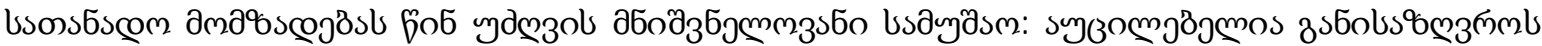

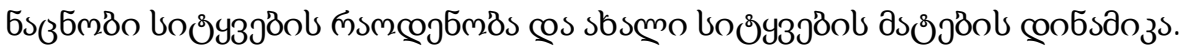

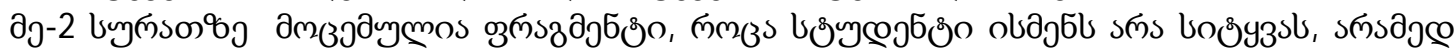

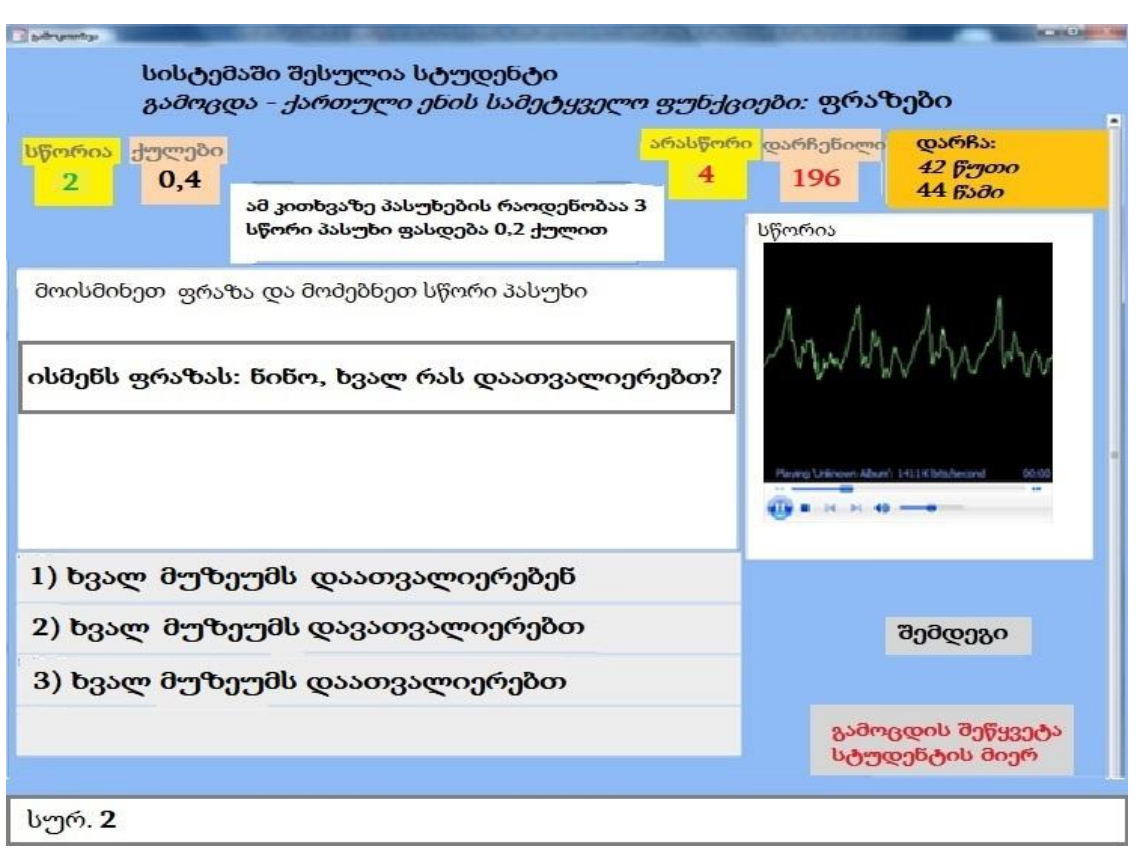
foбscoscojasl (sa

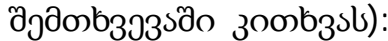
„бобм bзsm msls

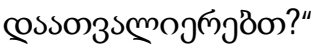

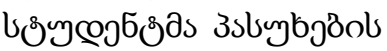

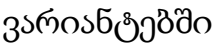

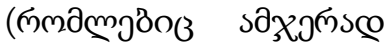
jง

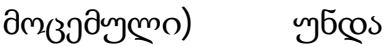
ambstbmb foбscoscojas,

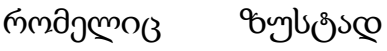
Әjjlsabajas coskaym

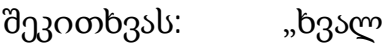
aybjyals

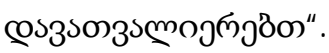

jl bsवysmmisls

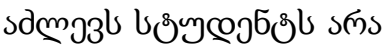




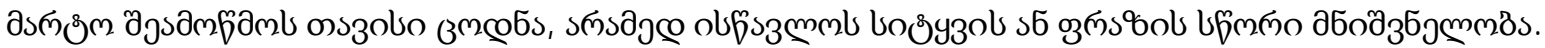

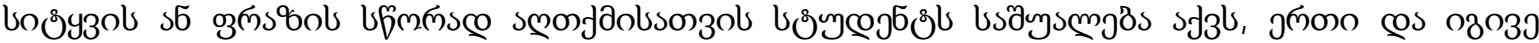

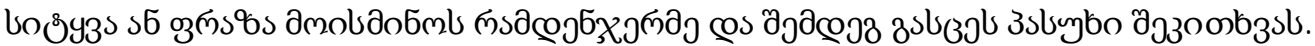

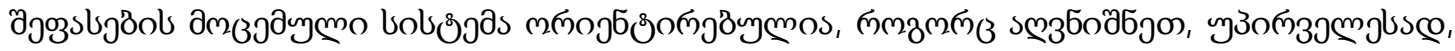

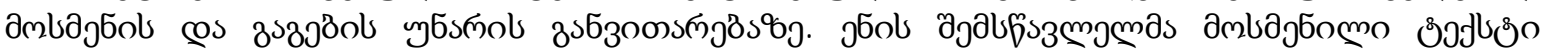

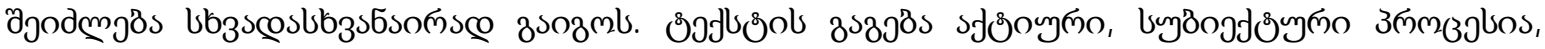

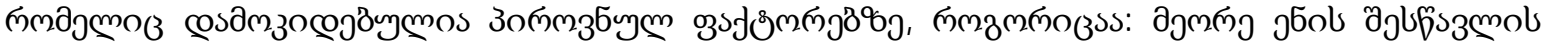

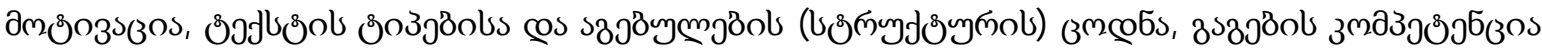

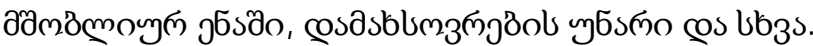

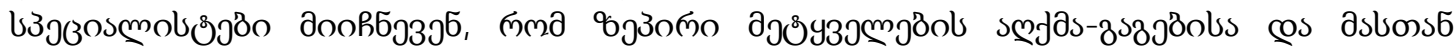

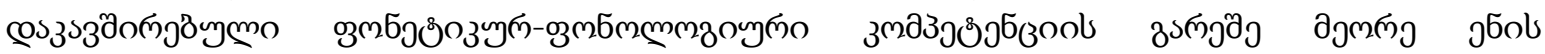

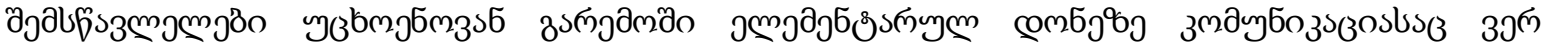

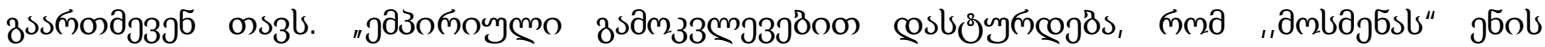

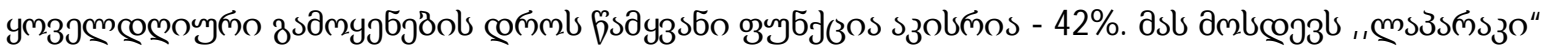

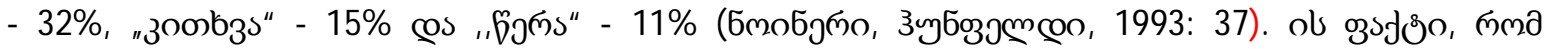

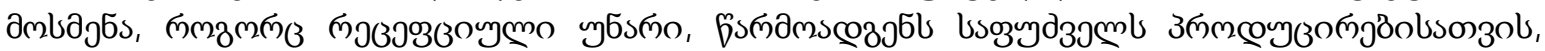

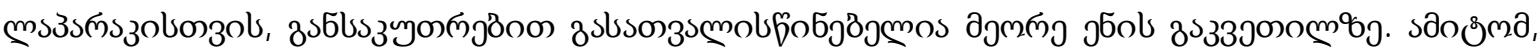

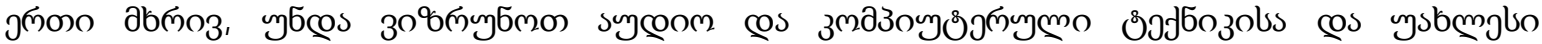

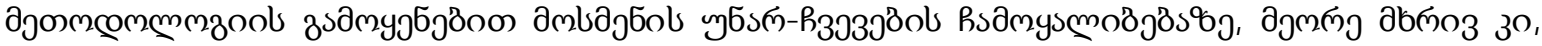

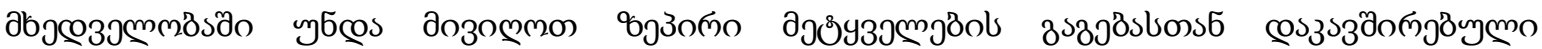

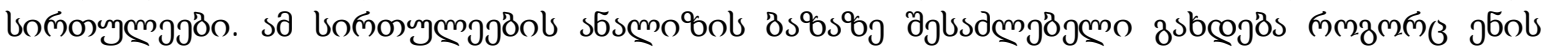

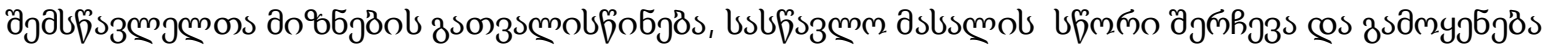

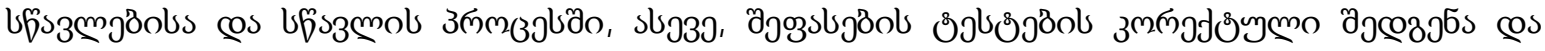

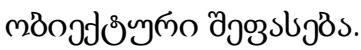

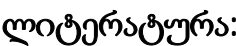

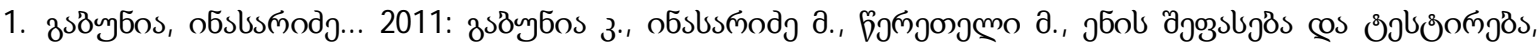
๓в̈omolo

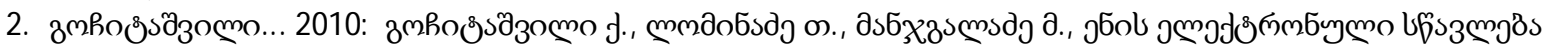

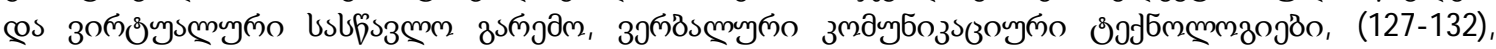
ỏnomolo

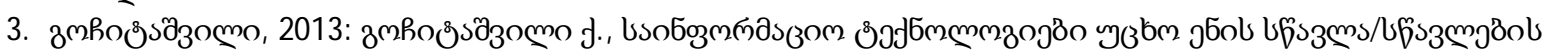

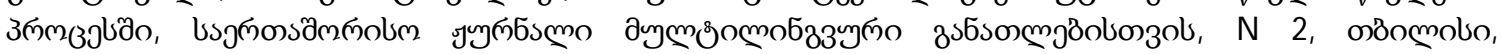
www.multilingualeducation.org 


\title{
Some Issues of Electronic Teaching and Assessment System in Non-Georgian Speaking Groups
}

Chokharadze Mariam

Shota Rustaveli State University, Batumi

\begin{abstract}
Assessment plays an important role in the planning and implementation process of teaching. Assessment is a continuous process of collecting, recording and analyzing quality data to achieve learning objectives, a consistent study of the success and progress of language learners.

In this paper we discuss the issues of teaching and assessment of Georgian as a foreign language. First of all, we will discuss some strategies related to learning process. We will present our innovation in the assessment system. This is a novelty that we have worked on with our colleagues at Batumi Shota Rustaveli State University within the short-term educational program "Georgian Language for Foreigners".

In this work we will discuss about how to use the electronic resources to develop specific language competencies and assess the knowledge based on relevant materials. We note that the article mainly reflects our personal experience, which we have accumulated in the teaching process, the innovations that we have tried to introduce to groups of non-Georgian listeners.
\end{abstract}

Keywords: teaching, assessment, listening, understanding, comprehension. 